\title{
Research on Visualization model of IT Operations Management System
}

\author{
Hong Xia ${ }^{1, a}$, Fei $\mathrm{Wu}^{1, \mathrm{~b},{ }^{*}}$ \\ ${ }^{1}$ North China Electric Power University Beijing, Beijing, China \\ asummerday@ncepu.edu.cn, bwufeichn@126.com \\ ${ }^{\star}$ Fei $\mathrm{Wu}$
}

\begin{abstract}
Keywords: IT business system, IT operations management, Zabbix, IT Service Management.
Abstract. With the rapid development of information technology, IT business system is gradually increasing, and business system more and more depends on the information platform. Information platforms need a large number of IT elements support, monitoring software has become a key part of this. Whether relying on purely artificial or using a variety of IT operations management software for IT operations management, it can not meet the actual needs, and will give the IT operations management department to bring a lot of manpower, material and time consumption. This paper combines the mainstream IT operations software Zabbix, studied the status and shortcomings of the traditional IT operations management system, and finally through the IT operations management process theory and application model research, combined with IT service management (ITSM) proposed visualization model of IT operations management system to improve the efficiency of IT operations management.
\end{abstract}

\section{Introduction}

With the rapid development of information technology, IT business system construction is increasing more than $30 \%$ year by year. In order to be running stably to meet the business needs, put a lot of new equipment, new technology: blade servers, storage and so on. In the IT system construction, usually according to the use of different equipment manufacturers, the construction cycle is different, and choose to monitor products or use some open source products, such as IT systems in the network equipment, the use of network equipment monitoring software, such as Cisco Monitoring software can only monitor Cisco equipment, such monitoring software can not monitor other products, it is only a single brand of network management software. At present, the mainstream IT operations management software such as Zabbix, Nagios, Ganglia and other products on the basic monitoring of IT elements have been relatively perfect, but in the IT and business integration, change management, problem management and many other aspects of the process are still very little, it can not meet the current needs of IT operations management.

\section{The status of IT operations management}

\subsection{Main problems in the IT operations management process}

The current business system is increasingly dependent on the information platform, this information platform is not only composed of networks and links, but also the server, database, storage and many other IT elements of support, so it need to use a lot of software to work, or rely on pure artificial maintenance. In the case of using a variety of IT operations management software, the network is very complicated, and this is the management islanding phenomenon. To the IT operations management department has brought a lot of manpower, material resources, time consumption.

To sum up, in the absence of comprehensive monitoring of IT systems, there are many problems:

1. Through the IT operations management engineers regularly log on the device or use a number of monitoring software to view the operation, the workload is large, and the content is not very detailed. 
2. Finding problem is not active, basically rely on the user phone reported failure, engineers and then troubleshooting.

3. IT management and business management out of touch, user reported the problem which is often a business system problems, and engineers can only see the equipment failure, not from the user's point of view to the business point of failure, resulting the need to coordinate the network, systems, databases, middleware and other IT operations management personnel together to find the fault point.

4. How to establish an effective IT operations management system, to build their own IT service management system, so that the IT operations management standardization, information transparency.

\subsection{The introduction of IT Service Management (ITSM)}

IT Service Management (ITSM) is a set of methodology that helps companies manage IT systems directly, manage, develop, implement and operate. ITSM originated from ITIL (IT Infrastructure Library, IT Infrastructure Library), ITIL is CCTA (British National Computer Bureau) in 1980 developed a set of IT service management standard library. It sums up the UK's approach to IT management and becomes a normative approach to providing a set of standard methods for planning, developing, implementing and maintaining operations for IT departments. Architecture framework of ITSM is shown in Fig. 1.

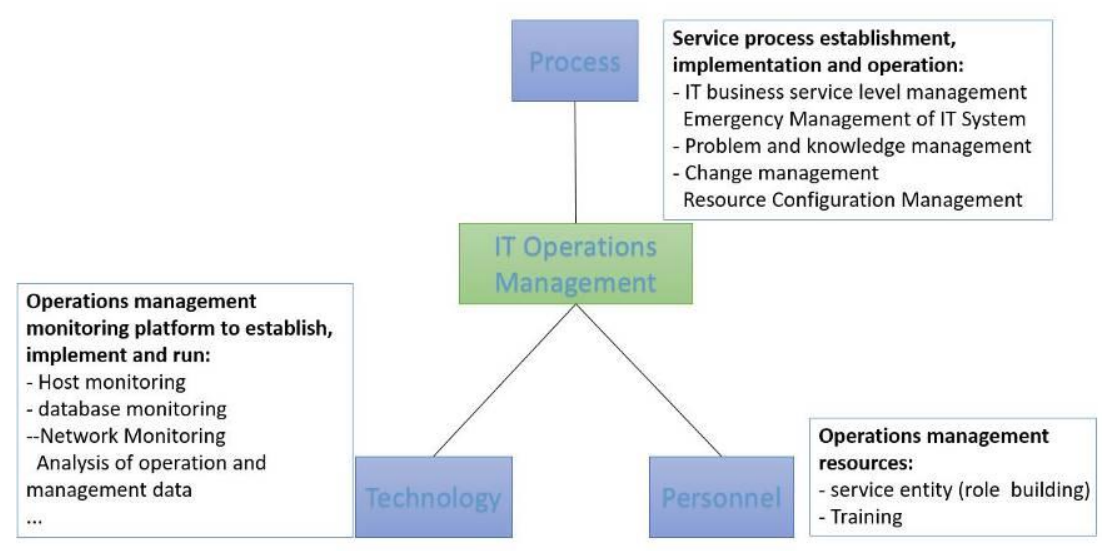

Fig. 1. Architecture framework of ITSM

ITSM is only a set of methodology, its final implementation relies on the appropriate tools and experience. As the domestic information is still in its infancy, so more attention to technology, for example, many customers also use the network management, system management and other management tools, but the technology only ensures the quality and efficiency of the service. The standard process is responsible for monitoring IT service operation, and personnel quality is related to the quality of service. ITSM is the most important of the process, personnel and technology of the three elements of the organic combination, ITSM in the implementation process not only the deployment of the corresponding management tools, and will be based on the specific circumstances of the development of personnel positions responsibilities, design daily work processes, and sudden Incident events and problem management processes.

\section{IT operations objectives}

\subsection{Comprehensive real-time monitoring}

It achieves the multi-vendor multi-technology level of unified monitoring of IT business platform for, including: host equipment, network equipment, storage devices, database management, middleware, application software and business systems, key operational indicators. 


\subsection{Flexible definition of business-to-IT association view}

IT operations management system program can establish a "business view" to reflect the health of the IT environment to the impact of the business, to protect the normal operation of the entire IT business platform.

$>$ Combine the complex IT infrastructure with the main line of business, and establish a vertical topology view of each business leading to the IT infrastructure monitoring point. For example, any one of the normal operation of the business needs network port, host, application software, business systems to work together. The goal of the IT operations management system is to focus and link all relevant technical aspects to establish a multi-level association of business logic.

$>$ The business topology view can plan multi-level sub-views according to the distribution area of the device, the business system type and the device type.

$>$ Once the business topology view is established, it should be able to support the flexible configuration modification interface so that the service management view can be quickly synchronized with the construction and adjustment of the IT business platform.

\subsection{To achieve a unified concentration of alarm management}

On the basis of comprehensive monitoring, establishing a unified central alarm classification rules, alarm correlation analysis rules, alarm notification rules, event response and processing of standardized work processes, to achieve system-wide alarm statistics and assessment.

\subsection{Hierarchical multiuser management}

Based on centralized monitoring data, alarm data, view data and various management functions, IT operations management system will provide managers with different roles to provide flexible user rights definition and management domain definition, clear division of responsibilities, shielding irrelevant information, meet Each administrator's personalized management needs.

IT operations management system to provide a sound user and authority management mechanism. The administrator defines different roles to provide completely different view filtering, alarm filtering and operational functions for different roles.

\section{Zabbix analysis}

Zabbix is an enterprise-class open source solution that provides distributed system monitoring and network monitoring based on the web interface. Zabbix can monitor a variety of network parameters to ensure the safe operation of the server system, and provide a flexible notification mechanism to allow system administrators to quickly locate / solve the existence of various problems. Zabbix working flow is shown in Fig. 2.

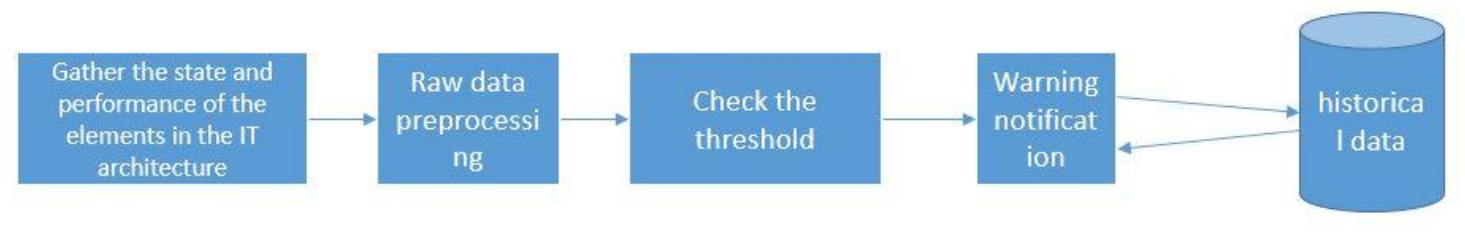

Fig. 2. Zabbix working flow

\subsection{Design of visual IT operations platform based on zabbxi and ITSM}

Zabbix to achieve the basis of IT element monitoring, a more comprehensive, but it is not directly related to IT business, so the door through the ITSM concept into zabbix, for example, when the network administrator found that the office server database server problems, IT Network administrators may only think that some of the conventional database problems, but this time the entire network office system has been completely paralyzed. If this problem, from the business point 
of view of the management, network administrators as long as the monitoring of the overall business system can view the office, when the office system in any one server problems, the first administrator and salesman can see the whole Office system view in the alarm, but also through the view quickly and intuitively find the root cause of the problem. Office system business association is shown in Fig. 3.

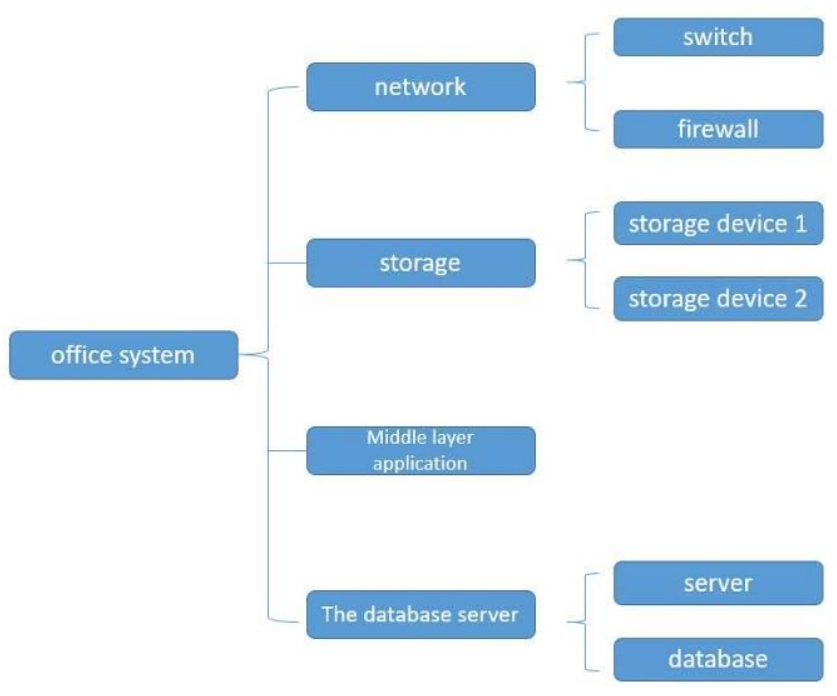

Fig. 3. Office system business association

In view of the current situation of IT operations management, this paper puts forward IT operations management system platform model, the platform model consists of two parts:

1. person-oriented / process part.

2. for the IT environment part.

First of all, IT environment is part of the IT operations management monitoring platform to achieve all the elements of IT environment to conduct a comprehensive monitoring, including: network equipment, servers, applications, databases and storage, etc., and can be Integration with a variety of existing management software.Through this one platform to achieve the IT environment management, data unified monitoring. IT operations management system platform model is shown in Fig. 4.
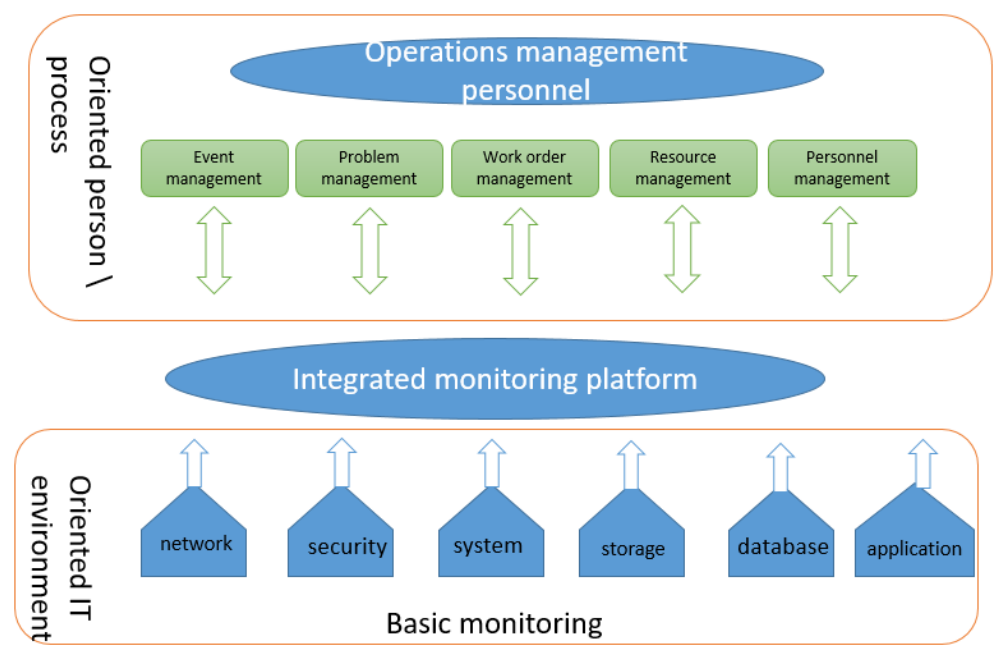

Fig. 4. IT operations management system platform model

\section{Summary}

The monitoring scope of IT operations management system platform model includes: network switches, routers, firewalls, servers, databases, operating systems, middleware, software, services, 
websites, processes, ports and log files. Introduce ITSM, IT business to guide the management of IT resources and performance, so that managers really see the operation of each IT business status, and through the IT business view, quickly find the key elements which makes IT business can not run. Through the business view configuration, the management model of the customer service can be defined flexibly, and the association of the managers from the macroscopic to the microscopic, from the business logic layer to the technical level of the device is realized, it realizes the real service-oriented visualization platform monitoring.

\section{References}

[1] Wan, Stewart H. C., and Y. Chan. "Improving service management in campus IT operations." Campus-Wide Information Systems 25.1(2008):30-49.

[2] Gupta, Chetan. "Event correlation for operations management of largescale IT systems." International Conference on Autonomic Computing 2012:91-96.

[3] Rai, Veerendra K, and P. Chandak. "Shift planning and scheduling for IT service operations management." Systems Conference IEEE, 2015:645-652.

[4] Wan, Stewart H. C., and Y. Chan. "Improving service management in outsourced IT operations." Journal of Facilities Management 5.3(2007):188-204.

[5] Gupta, Rajeev, K. H. Prasad, and M. Mohania. "Automating ITSM Incident Management Process." International Conference on Autonomic Computing IEEE, 2008:141-150.

[6] Morana, Stefan, T. Gerards, and A. Maedche. "ITSM Process Guide - A Process Guidance System for IT Service Management." Desrist 2015:406-410.

[7] Weng, Liang, and B. Weng. "Research on Enterprise ITSM Knowledge Management Model." International Conference on Management and Service Science IEEE, 2009:1-4.

[8] Yao, Zhong, and X. Wang. "An ITIL based ITSM practice: A case study of steel manufacturing enterprise." International Conference on Service Systems and Service Management IEEE, 2010:1-5.

[9] Agranoff, Robert, and M. Mcguire. "Big Questions in Public Network Management Research." Journal of Public Administration Research and Theory: J-PART 11.3(2001):295-326.

[10]Kim, Hyojoon, and N. Feamster. "Improving network management with software defined networking." IEEE Communications Magazine 51.2(2013):114-119.

[11]Telesca, A, et al. "System performance monitoring of the ALICE Data Acquisition System with Zabbix." Journal of Physics Conference Series Journal of Physics Conference Series, 2014:062046.

[12]Aleksieva, Veneta Panajotova, Здравко Митев, and Сияна Вълчанова. "Personal Monitoring and Managenent of Virtual Infrastructures based on Zabbix." Ru\&su 2016. 